\title{
Pneumatic Structure with Kinetic Sub-system: A Proposal for Extraterrestrial Life
}

\author{
Asli Agirbas \\ Fatih Sultan Mehmet Vakif University, Istanbul | Turkey | asliagirbas@gmail.com
}

\begin{abstract}
Designing for extraterrestrial life is a very up-to-date issue. However, there are many constraints in this kind of designs. Designs that provide the best solution can only be obtained by identifying these constraints very well. In this study, a design concept was developed for life in Mars by considering various constraints. This design consists of a kinetic system with pneumatic structure. The preliminary scheme of this structure, which was planned to produce as a prototype, was discussed in the scope of this study.
\end{abstract}

Keywords: Extraterrestrial architecture; Martian base; Pneumatic structure; Kinetic structures; Algorithmic and parametric design

\section{INTRODUCTION}

As Zubrin (2014) says, future colonies can be created in extraterrestrial areas. Today, the dates of extraterrestrial life-focused missions to other planets are defined with governments and private companies like SpaceX. In addition, various architectural competitions are being organized on how to colonize in these extraterrestrial fields. For example, NASA has launched a design competition entitled "3D printed Habitat Challenge," awaiting the creation of innovative solutions for the housing of explorers in future missions to the Moon, Mars and beyond. The platform named "Mars City Design" opens competition for Martian architecture every year since 2016. The platform "Eleven Magazine" opened Moontopia (2016) competition related to Lunar architecture and Marstopia (2018) competition related to Martian architecture. In 2018, UIA opened the competition entitled "The Capsules of Extraterrestrial Life" for the participation of young architects. In many student studies, extraterrestrial life oriented projects are being developed (Leach, 2014). Therefore, in today's contemporary architecture world, studies on design for extraterrestrial areas have begun to be produced.

There are too many constraints for designs to be made in extraterrestrial areas. For example, if we consider designing a space for living on Mars, we must take into account the following problems: the logistic problem, problems to be experienced in the construction process, the production of energy problem, the radiation problem, the lack of gravity, and the unbreathable atmosphere (Benaroya et. al., 2002; Kozicka, 2008; Kozicki and Kozicka, 2011; Morris et. al., 2016).

In this study, a proposal of a structure for Mars was made with taking into account the above mentioned problems.
This proposed structure is a pneumatic structure and has kinetic sub-system.

Kinetic structures (Zuk, 1970; Senagala, 2005) and pneumatic structures are widely studied. Biloria and Oosterhuis (2005) and Ahlquist et. al. (2017)'s studies on pneumatic structure, and Abdelmohsen et. al. (2016) and Khoo et.al (2011)'s studies on kinetic structure can be given as examples. But, particularly for extraterrestrial fields, design-oriented studies on this subject are limited.

This study focuses on the first stage of planned prototype to be produced. In this stage, the possible emerging problems in extraterrestrial life and their solutions and the conceptual design were described throughout a case study.

\section{METHODOLOGY}

While performing the experiment of the structure in this work, the Grasshopper program, which is a plug-in to the Rhino program, and Kangaroo add-on were used. Kangaroo add-on's 'mesh pressure' component was used to construct the pneumatic structure created according to the kinetic sub-structure, while the 'origami' component was used to construct the kinetic sub-structure. Weaverbird add-on was also used to make mesh to line, line to mesh transitions (Figure 1).

For this, one of the selected Ron Resch's folding patterns was considered as a kinetic structure. This folding pattern was selected because it can take a dome form. Thus, closed form can be created easily. Then, this triangular modular kinetic system was used as the infrastructure of the pneumatic structure (Figure 2). 


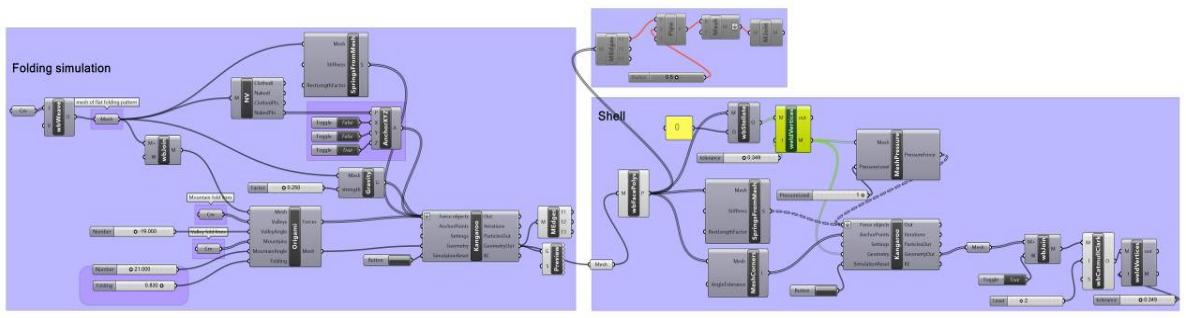

Figure 1: The algorithmic infrastructure of the 'Pneumatic Structure with Kinetic Sub-system' model

Related experiments on the forms created by the kinetic sub-structure, and various experiments on the state of the pneumatic structure at various pressure values have been made (Figure 3, Figure 5). It is considered appropriate to use the scissor system for the connection of the structure (planned to be suspended in the air) to the ground.

As many examples can be found on the Internet, origami component, gravity component, anchorXYZ component and "springs from mesh" component were connected to "force object" input of Kangaroo physics engine. Mesh geometry, which was formed from the result of origami, was connected to the geometry input of Kangaroo physics engine. Geometry was determined by simulation. With the mesh geometry obtained as a result of this simulation, a second simulation was done.

This second simulation is related to the pneumatic surface formed on each surface of this form. The polylines on the mesh formed as a result of the first simulation were defined with "weaverbird's face polylines" component. These polylines were connected to the inputs of the components that were included in the second Kangaroo simulation. In the second simulation, "mesh pressure" component and "springs from mesh" component were connected to "force object" input of the Kangaroo physics engine. "Mesh corners" component was connected to the "anchor points" input of the Kangaroo physics engine. As a result of this second simulation, the pneumatic layer was formed. When creating this layer, different pressure level values can be given to "Mesh Pressure" component to differentiate the form.

\section{CONCEPTUAL DESIGN ELEMENTS}

In this proposal, we considered various constraints for living space in extraterrestrial life. According to the constraints, we found that the living space must have a lightweight structure. It should include self-construction process. It should protect person from the radiation. It should also have basic spatial organisation.

\section{LIGHTWEIGHT-STRUCTURE}

Portable, reusable and adaptable lightweight-structure was formed by pneumatic system. This material can easily be transferred from Earth to Mars since it occupies little space (Figure 2, Figure 3).

Pneumatic Structure with Kinetic Sub-System will be fast and adaptable system since it uses minimum material, it is made on site, the logistic is reduced to minimum and it is reusable.

\section{SELF-CONSTRUCTION PROCESS}

In order not to transfer the kinetic sub-system from Earth to Mars, the production on Mars with local material was considered to be appropriate.

For this, autonomous robots are thought to construct a kinetic sub-system, using 3D printing technique and

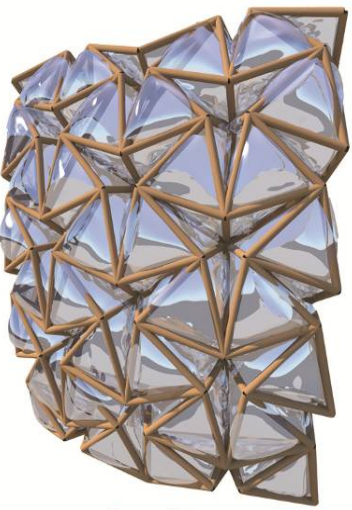

Pneumatic Layer

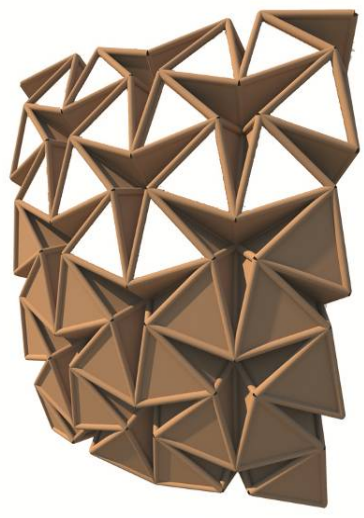

Solid-Void Layer

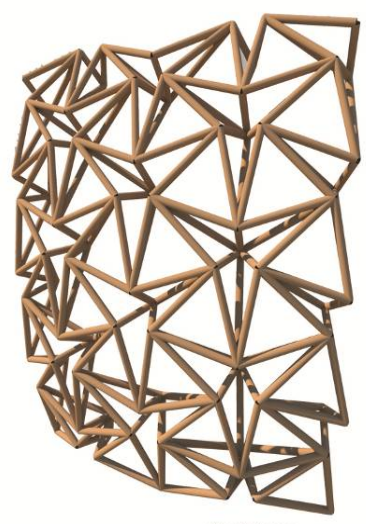

Kinetic Layer

Figure 2: Layers of the 'Pneumatic Structure with Kinetic Sub-system' model 
Martian soil. As Wilkonson et. al. (2016) proposed in design studies for Mars, artificial intelligence based robots can be used in construction.

These robots are able to produce solutions to the problems that arise during the construction process. All robots with self-organizing logic like swarm intelligence (Agirbas, 2019) can continue to work. If any robot breaks down, the other robot can take over the job.
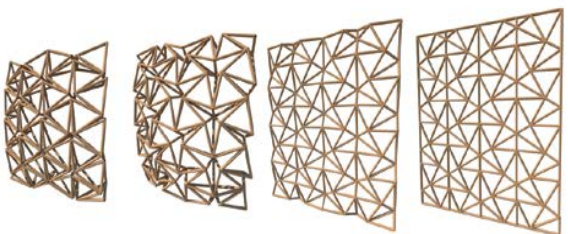

Partial Folding Pattern of Kinetic Layer (Energy distribution system)
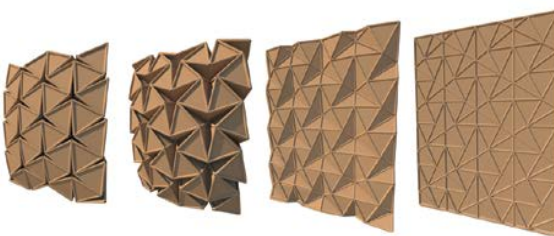

Partial Folding Pattern of Solid-Void Layer (Energy collector and radiation protector system)
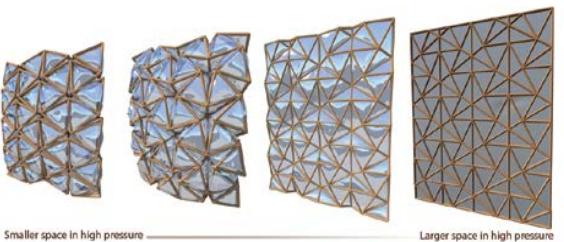

Partial Folding Pattern of Pneumatic Layer

Figure 3: Forms created with folding

\section{PHOTOVOLTAIC TEXTILES}

Nowadays, works on Photovoltaic Textiles (Mather and Wilson, 2017) are being done. So, it was thought that energy could be collected by the photovoltaic textile, which will be placed underneath the pneumatic layer. Energy will be stored in the kinetic sub-system and the necessary energy will be distributed from there (Figure 4).

During the sunlight reception times, protection from the radiation on Mars may also be provided with photovoltaic textile covers. At the same time, photovoltaic textile (function as solar panel) placed on these covers will collect sunlight and provide energy production. In addition, this photovoltaic textile cover will form the desired solidvoid ratio on the facade.

\section{SPACE ORGANISATION}

With the kinetic sub-system, folding units can be obtained. Thus, it is possible to make separate spaces such as private space, common space. Also, a closed space is created to provide a breathable and clean system (Figure 5, Figure 6, Figure 7). With a smart system, many space configurations can be achieved by folding typology (such as sphere-like or pillow-like forms).

With the Pneumatic Structure having Kinetic Sub-System, the balance of air pressure can be provided in the space. In accordance with volume-pressure relationship, the pressure can be adjusted via increasing or decreasing the volume with Kinetic sub-system.

Space organization has effects on human psychology. However, many factors other than space organization can also affect human psychology in extraterrestrial life. This subject is also an important subject to work on. The studies on this subject are done on the axis of psychology-extraterrestrial life, as Seguin (2005) did.

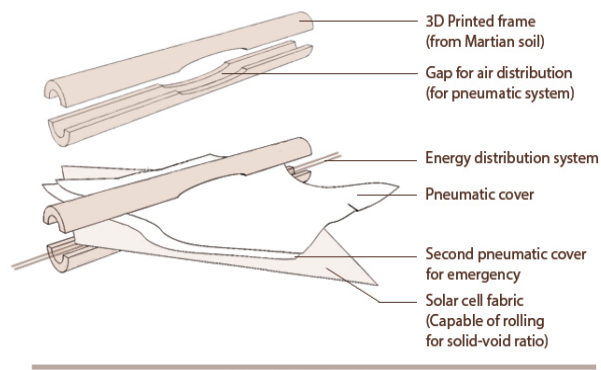

Detail of the frame

Figure 4: Detail of the frame with photovoltaic texiles

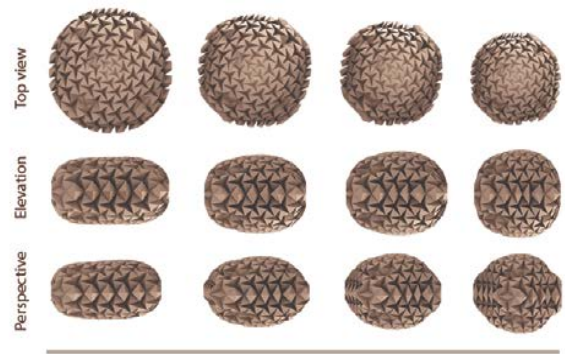

Sphere-like Folding Process

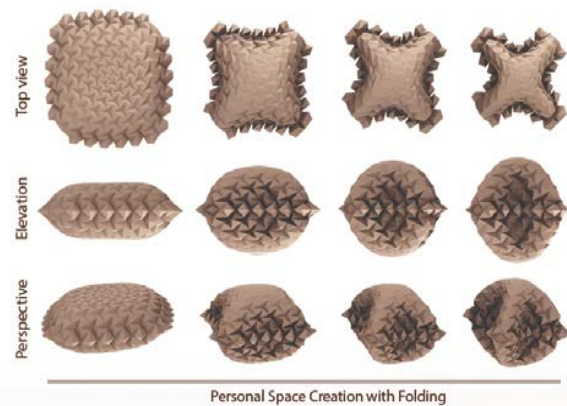

Figure 5: Different folding typologies 

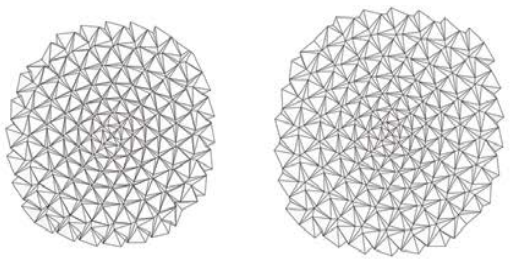

$\rightarrow<$

Figure 6: Folding process

\section{SYSTEM AS HABITAT}

Due to the lack of sources (water, $\mathrm{O}_{2}$ ) in the new habitat to be created, relevant storage and production units have to be created. In the same way, the waste must be managed.

Since these units need to be linked to other habitat units, the related infrastructure needs to be established. Various separate units for Greenhouse, Water storage, $\mathrm{O}_{2}$ storage and waste (such as excrement, dirty water, garbage) will be created and these units will be connected to the habitat units. In addition, a separate unit will be made for the recycling of wastes.

Infrastructure will also be established while connecting those with each other. The structure of the infrastructure will be configured as a scissor system. This system was created with the logic of other units (Pneumatic Structure with Kinetic Sub-System). Therefore, it carries the same features as the other units. At the same time, with the Scissor system, the units will be installed at the ground level. Thus, due to the lack of gravity, the units will remain suspended in the air. With the mechanical movement of the scissor system (shortening or extending), the units will also be able to change their places (Figure 8, Figure 9).
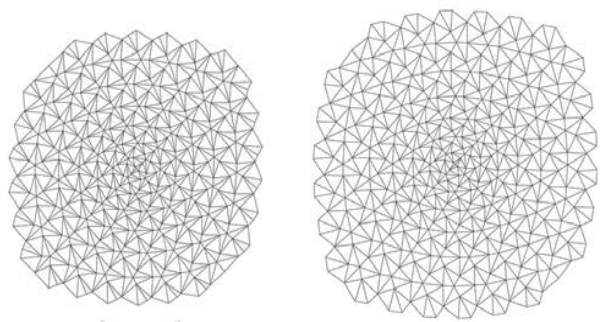

\section{CONCLUSION}

In this study, a conceptual design of a new structural system was made with the 'Pneumatic Structure with Kinetic Sub-system' model. The studies on such models are important both for the development of new structural systems and for the development of extraterrestrial habitat design alternatives, which is a very up-to-date issue.

The 'Pneumatic Structure with Kinetic Sub-system' model created in this study was developed using visual programming language. Thus, the generated script can be easily used and developed by other designers. Since the design of extraterrestrial habitats have many constraints, developable models will be more beneficial to the scientific community in terms of helping to develop better models.

Simulations are being generally used for building physics related subjects. For example, they are commonly used for daylighting analysis, energy analysis and optimizations. However, simulations have also a very important role in design process, because, they can simulate building form. As in this study, simulations are very important for creation of pneumatic structures. It is possible to see pressure-form relationship in the simulations.
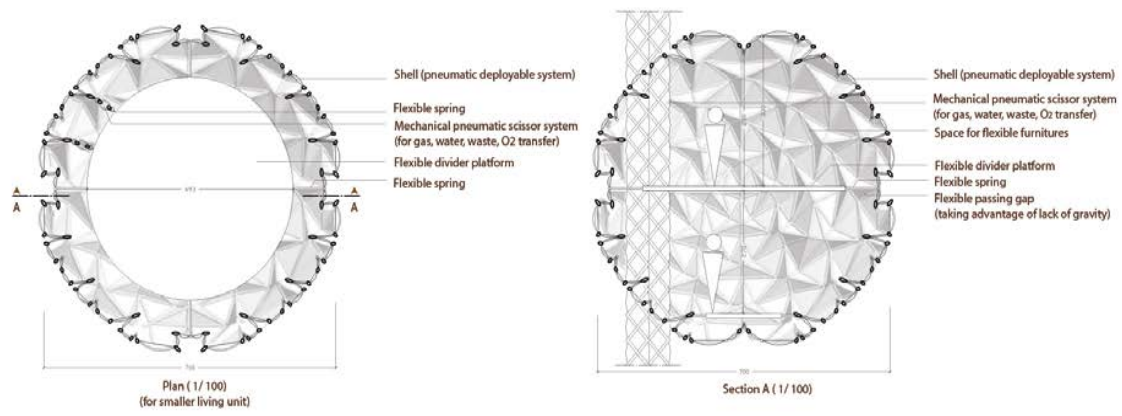

Figure 7: An example of space configuration 


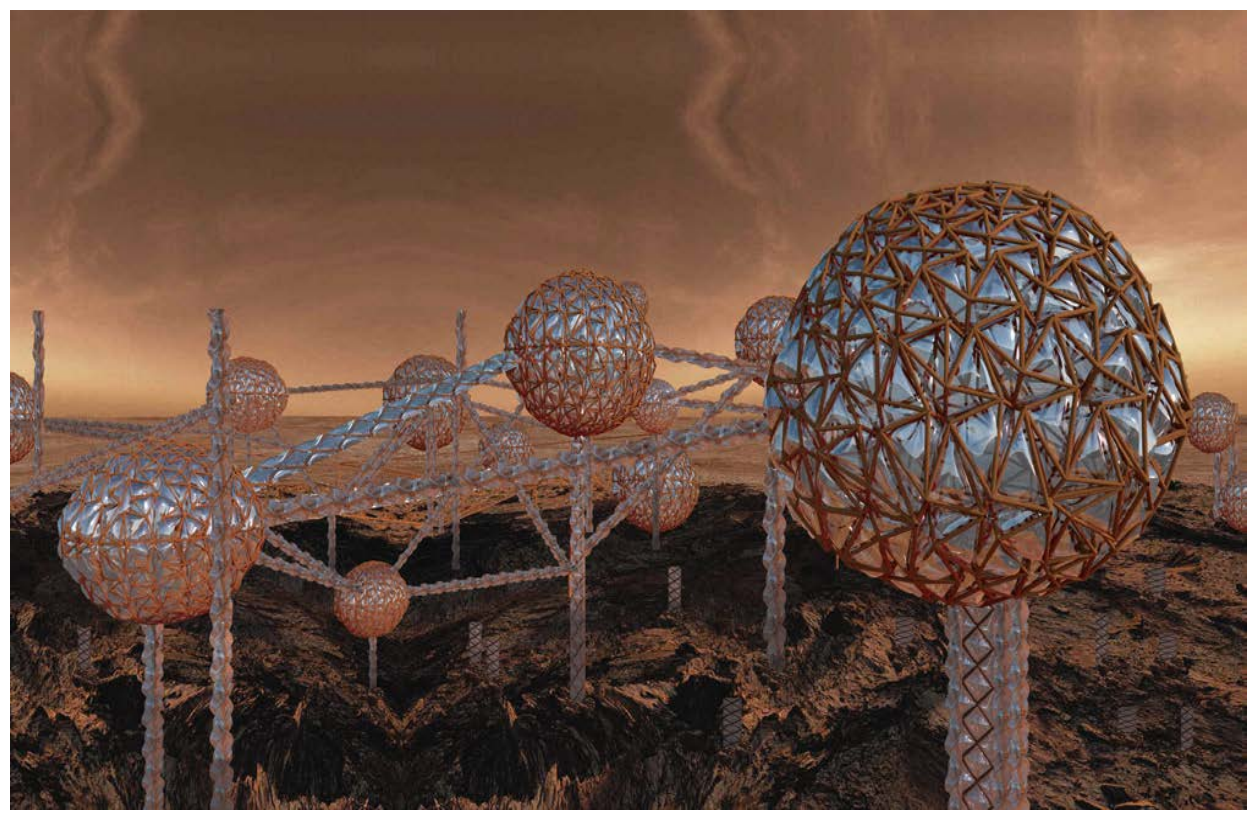

Figure 9: Senario of the habitat

The next step of this study is the development of a prototype of the developed conceptual model. Using this prototype, it is planned to measure the resistance of the kinetic pneumatic system at different pressure levels. In order to produce this prototype, the mechanism in the nodes of the kinetic system must first be solved. In addition, this kinetic system should be double-layer, then, pneumatic layer can be placed between the two layers. After selecting the appropriate material, the pneumatic layer can be placed in the double layer kinetic system. Then, with different pressure settings, form formation and durability can be measured in this system.
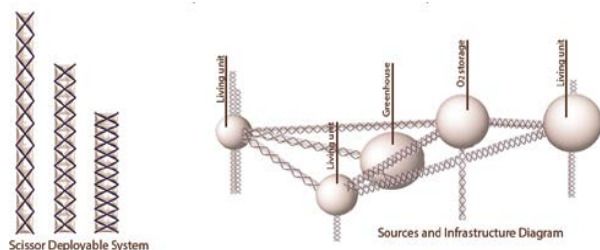

Figure 8: Senario of the system

It is also possible to use some of origami patterns for creating folding structures. There are many studies about the use of origami patterns for form-finding. Studies of Baerlecken et. al. (2012), Hemmerling (2010), Lee (2013), Lee and Leounis (2011) and Agirbas (2017) can be given as examples of this subject.

The used folding pattern in this study is based on Ron Resch's pattern. However, there are many folding pattern types which have different geometric characteristics. Different types of folding patterns can be used for pneumatic structure. Therefore, comparative research about this issue can be done.

\section{REFERENCES}

Abdelmohsen, S., Massoud, P. \& Elshafei, A. (2016). Using Tensegrity and Folding to Generate Soft Responsive Architectural Skins. In Proceedings of the 34th eCAADe Conference, University of Oulu, Oulu, Finland, 22-26 August (pp. 529-536).

Agirbas, A. (2017). The Use of Simulation for Creating Folding Structures: A Teaching Model. In Proceedings of the 35th eCAADe Conference, Sapienza University of Rome, Rome, Italy, 20-22 September (pp. 325-332).

Agirbas, A. (2019). Façade Form-Finding with Swarm Intelligence. Automation in Construction, 99, 140-151.

Ahlquist, S., McGee, W. \& Sharmin, S. (2017). PneumaKnit: Actuated Architectures Through Wale- and Course-Wise Tubular Knit-Constrained Pneumatic Systems. In Proceedings of the 37th Annual Conference of the Association for Computer Aided Design in Architecture (ACADIA), Cambridge, MA, 2-4 November (pp. 38-51).

Baerlecken, D., Swarts, M., Gentry, R. \& Wonoto, N. (2012). BioOrigami: Form Finding and Evaluation of Origami Structures. In Proceedings of the 30th eCAADe Conference, Prague,Czech Republic, 12-14 September (pp. 497-504).

Benaroya, H., Bernold, L. \& Meng Chua, K. (2002). Engineering, Design and Construction of Lunar Bases. Journal of Aerospace Engineering, 15(2), 33-45.

Biloria, N. \& Oosterhuis, K. (2005). Envisioning the RESPONSIVE Milieu: An Investigation into Aspects of Ambient Intelligence, Human Machine Symbiosis and Ubiquitous Computing for Developing a Generic Real-time Interactive Spatial Prototype. In Proceedings of the 10th International Conference on Computer-Aided Architectural Design Research in Asia (CAADRIA), New Delhi, India, 2830 April (pp. 421-432).

Hemmerling, M. (2010). Origamics. In Proceedings of the Arab Society for Computer Aided Architectural Design Conference (ASCAAD), Fez, Morocco, 19-21 October (pp. 89-96). 
Khoo, C.K., Salim, F. \& Burry, J. (2011). Designing Architectural Morphing Skins with Elastic Modular Systems. International Journal of Architectural Computing, 9(4), 397-419.

Kozicka, J. (2008). Low-cost Solutions for Martian Base. Advances in Space Research, 41, 129-137.

Kozicki, J. \& Kozicka, J. (2011). Human Friendly Architectural Design for a Small Martian Base. Advances in Space Research, 48, 1997-2004.

Leach, N. (2014). Projecting into Space: International Student Projects. Architectural Design, 84, 96-107.

Lee, D. (2013). Self-Organizing Origami Structures. In Proceedings of the 33rd Annual Conference of the Association for Computer Aided Design in Architecture (ACADIA), Cambridge, October 24-26 (pp. 421-422).

Lee, D.\& Leounis, B. (2011). Digital Origami: Modeling Planar Folding Structures. In ACADIA Regional Conference Proceedings, University of Nebraska Lincoln (pp.25-29).

Mather, R.R. \& Wilson, J.I.B. (2017). Fabrication of Photovoltaic Textiles. Coatings, 7(5), 63.

Morris, M., Ciardullo, C., Lents, K., Montes, J., Yashar, M., Rudakevych, O.,Sono, M. \& Sono, Y. (2016). Mars Ice
House: Using the Physics of Phase Change in 3D Printing a Habitat with $\mathrm{H} 2 \mathrm{O}$. In 46th International Conference on Environmental Systems, 10-14 July, Vienna, Austria.

Seguin A.M. (2005). Engaging Space: Extraterrestrial Architecture and the Human Psyche. Acta Astronautica, 56, 980-995.

Senagala, M. (2005). Building is a Network for Living in: Toward New Architectures. In Proceedings of the 2005 Annual Conference of the Association for Computer Aided Design in Architecture (ACADIA), Savannah (Georgia) 13-16 October (pp. 36-47).

Zuk, W. (1970). Kinetic Architecture. New York, NY: Van Nostrand Reinhold.

Zubrin, R. (2014). Colonising the Red Planet: Humans to Mars in Our Time. Architectural Design, 84, 46-53.

Wilkinson, S., Musil, J., Dierckx, J., Gallou, I. \& De Kestelier X. (2016). Autonomous Additive Construction on Mars. In Earth \& Space 2016 Conference - ASCE International Conference on Engineering, Science, Construction and Operations in Challenging Environments. 